\title{
Frictional heating calculations for polymers
}

\author{
M. Conte, B. Pinedo \& A. Igartua \\ IK4-Tekniker, Eibar, Spain
}

\begin{abstract}
In a previous study, the authors highlighted the importance of estimating flash temperature at the contact surface of a polymer rubbing against steel. Subsequently a calculation method based on experimental tests was developed. The aim of this work is the validation of such method on different kinds of polymers, such as Nitrile-Butadiene Rubber (NBR), Thermoplastic Polyurethane (TPU) and Polytetrafluoroethylene (PTFE). The characteristics of the materials are analyzed by means of Thermo Gravimetric (TGA) and Differential Scanning Calorimetry (DSC) analyses. Frictional tests are performed on an Optimol SRV tribometer in high frequency reciprocating motion. The viability of the calculation method and its limits are herein explained.

Keywords: polymers, friction, heating, wear, temperature rise.
\end{abstract}

\section{Introduction}

Temperature is one of the most limiting factors of polymers. When two bodies slide against each other, most of the energy dissipated during the process of friction is mainly converted into heat resulting in a local and usually consistent temperature rise. This phenomenon is known as frictional heating. This temperature variation on service has influences on the physical and chemical ageing, producing changes in mechanical and thermal properties, that is, thermal degradation, thermal expansion and structural changes in polymers. In order to satisfy the requests of the market, which is continuously demanding for high performance materials, there is a need to study the behaviour of polymers, the wear mechanisms developed and surface temperatures reached during sliding.

In a previous study, the authors highlighted the importance of estimating flash temperature at the contact surface of a polymer rubbing against steel (Conte et al. [1]). Subsequently a calculation method based on experimental tests was developed using Tian and Kennedy's equations (Conte et al. [2]). The aim of this 
work is the validation of such method on different kind of polymers, such as Nitrile-Butadiene Rubber (NBR), Thermoplastic Polyurethane (TPU) and Polytetrafluoroethylene (PTFE). These materials were chosen because of their different nature and because of their wide use in the industry for manufacturing components, such as seals. NBR is a thermoset polymer that has a good resistance to oils, good adhesion to metals, moderate friction coefficient and moderate thermal resistance (up to about $110^{\circ} \mathrm{C}$ ). TPU is a thermoplastic elastomer that combines the mechanical properties of the rubber with the processability of thermoplastics. It usually has a good wear resistance and good load capacity, a moderate service temperature range (up to about $110^{\circ} \mathrm{C}$ ) and sensitive to humidity. PTFE is a semi-crystalline thermoplastic material that presents a low friction coefficient even in dry conditions, a low carrying capacity, a high thermal resistance (its melting point is about $330^{\circ} \mathrm{C}$ ) and an excellent surface lubricity.

In the present study maximum contact temperature during sliding is calculated based on experiments considering the work done by the friction force (Conte et al. [2]). The polymeric samples are analyzed by means of Optical microscopy and Scanning Electron Microscopy (SEM) in order to investigate worn surfaces and identify dominant failure mechanisms. The frictional heating itself, in fact, could lead to structural changes and physical and chemical reactions on material surfaces to be re-conduced to contact temperature (Friedrich [3]).

\section{Experimental setup and specimens}

Friction and wear tests were carried out in the test rig represented in Figure 1: the polymeric samples (1) were mounted on a frame (2) and loaded by an axially sliding cylinder of AISI 52100 of $15 \mathrm{~mm}$ diameter and $22 \mathrm{~mm}$ length (3),
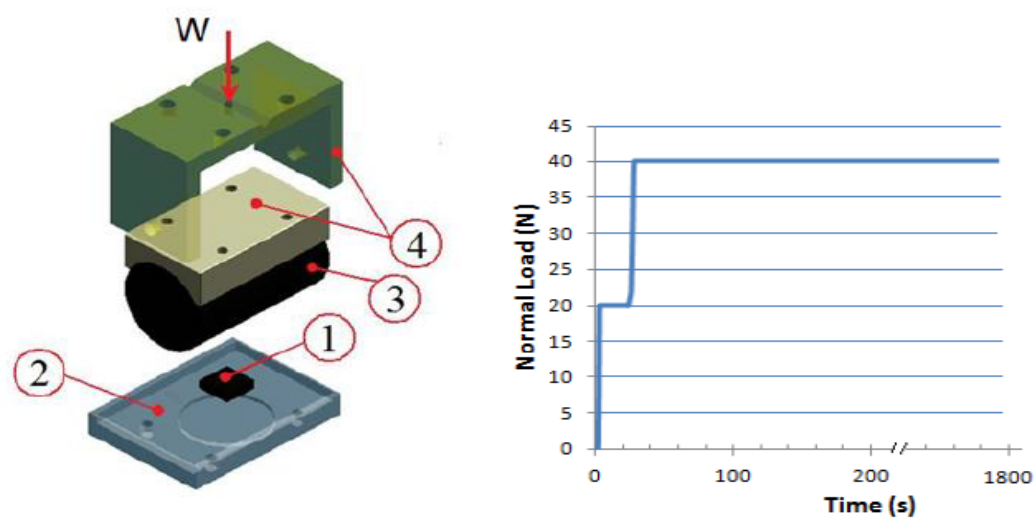

Figure 1: Test rig configuration: polymeric sample (1), frame (2), sliding rod (3), holders (4). Normal load is set on $20 \mathrm{~N}$ for the first $20 \mathrm{~s}$ and then at $40 \mathrm{~N}$. 
supported and fixed by two holders (4), on which normal constant load W is applied. The holders and a couple of screws avoid rotation of the rod around its axel. The system is located in a climate chamber in order to set temperature and humidity percentage. Normal load was set on $20 \mathrm{~N}$ for the first $20 \mathrm{~s}$ and then at $40 \mathrm{~N}$, with $70 \mathrm{~Hz}$ frequency in $2 \mathrm{~mm}$ stroke. Coefficient of friction (CoF) was recorded for 30 minutes; each test was repeated 2-3 times.

Table 1 shows some thermal and mechanical properties of the polymeric samples.

Table 1: $\quad$ Polymers' characteristics.

\begin{tabular}{|l|c|c|c|c|c|c|}
\hline Sample & $\begin{array}{c}\text { Thermal } \\
\text { conductivity } \\
\mathrm{K}(\mathrm{W} / \mathrm{mK})\end{array}$ & $\begin{array}{c}\text { Thermal } \\
\text { diffusivity } \\
\chi\left(\mathrm{m}^{2} / \mathrm{s}\right)\end{array}$ & $\begin{array}{c}\text { Melting } \\
\text { Temp. } \\
\mathrm{T}_{\mathrm{m}}\left({ }^{\circ} \mathrm{C}\right)\end{array}$ & $\begin{array}{c}\text { Heat of } \\
\text { fusion } \\
(\mathrm{J} / \mathrm{g})\end{array}$ & $\begin{array}{c}\mathrm{E} \\
(\mathrm{KJ})\end{array}$ & Hardness \\
\hline NBR & 0.24 & $1.48 \mathrm{e}^{-7}$ & $/$ & $/$ & 14.4 & 83 Sh A \\
\hline TPU & 0.25 & $1.20 \mathrm{e}^{-4}$ & 359 & $/$ & 21.9 & 96 Sh A \\
\hline $\begin{array}{l}\text { PTFE } \\
\text { Virgin }\end{array}$ & 0.24 & $9.24 \mathrm{e}^{-8}$ & 335 & 35.20 & 9.3 & 63 Sh D \\
\hline
\end{tabular}

For polymer characterization both thermal analysis techniques and microscopic analysis were used. Thermogravimetric Analysis (TGA) was used for studying their stability evolution with temperature. The curves in Figure 2 show the polymers degradation along temperature. The PTFE is thermically the most stable polymer, in fact, while the NBR and the TPU samples at temperatures around $200-250^{\circ} \mathrm{C}$ have already lost the $1 \%$ of its weight, the PTFE sample does not lose its weight until reaching approximately $500^{\circ} \mathrm{C}$. Weight losses are usually related to chemical reactions and/or physical transitions.

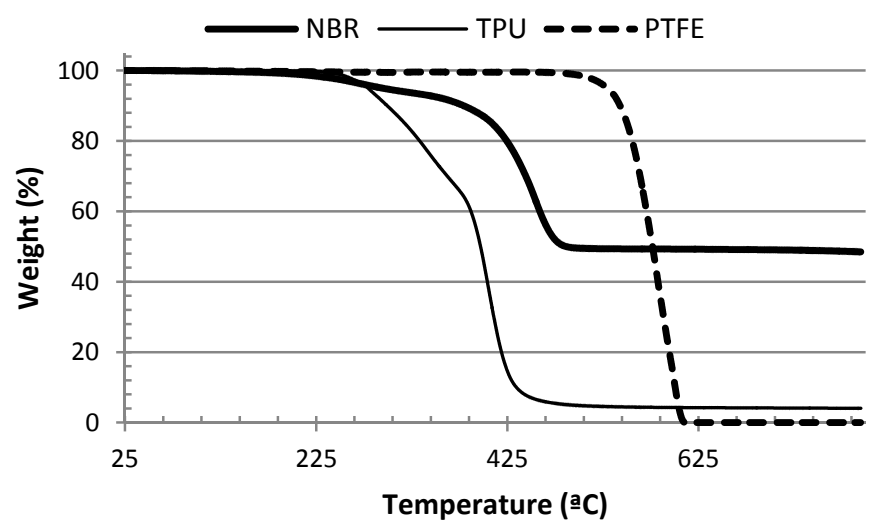

Figure 2: Thermal stability curves of polymeric samples with temperature studied by means of TGA analysis. 
Differential Scanning Calorimetry (DSC) was used to study the thermal transition of polymers. DSC analysis results of the polymers are shown in Figure 3. The NBR is a thermoset elastomer so that it does not melt at any temperature; it does not soften but decomposes on heating. TPU is a thermoplastic i.e. it can be reshaped by application of both heat and pressure. The peak at low temperature (around $100^{\circ} \mathrm{C}$ ) is related to the additives of the material whereas the peak at higher temperature (around $355^{\circ} \mathrm{C}$ ) is related to the disordering of the hard segments crystallites. The PTFE is a semi-crystalline thermoplastic which presents a melting point at approximately $335^{\circ} \mathrm{C}$ and a heat of fusion of $35.2 \mathrm{~J} / \mathrm{g}$. The heat of fusion is the ratio between the total heat input, that is, the area between the melting peak and the linear extrapolation of the curve after the glass transition, and the mass of the sample. It represents the amount of heat absorbed per unit mass of the material during melting.

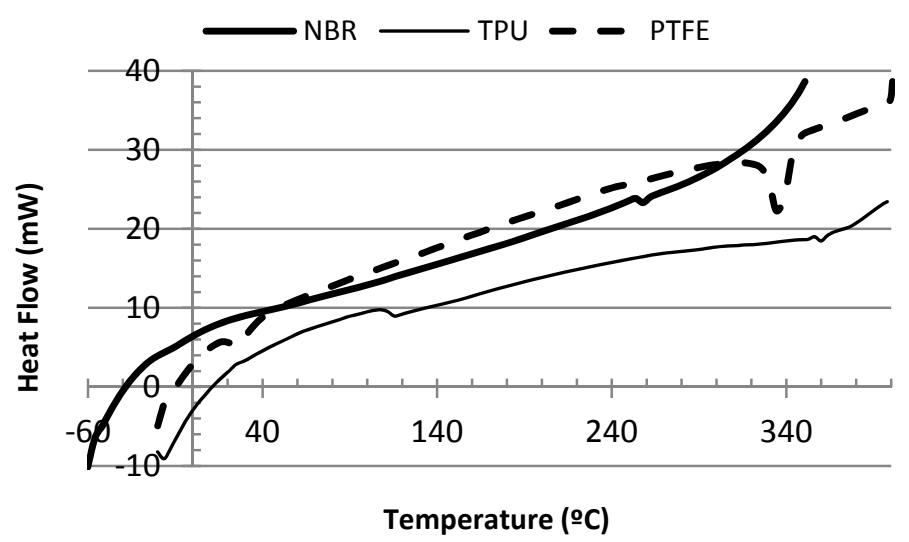

Figure 3: Thermal transition of samples under study and corresponding melting temperatures.

Before the frictional tests, Optical microscopy and Scanning Electron Microscopy (SEM) was used for investigating the worn surface. Figure 4 shows the samples surfaces before the tribo-tests. The measurements were repeated after the tribo-tests in order to appreciate eventual changes due to frictional heating.

\section{Tribological tests and frictional heating calculations}

In order to analytically estimate the flash temperature evolution during sliding, the following assumptions were taken: a linear contact area as a thermal source; all the generated heat is conducted into the sliding bodies; a steady state conductive heating thanks to the short stroke of the sliding movement in 


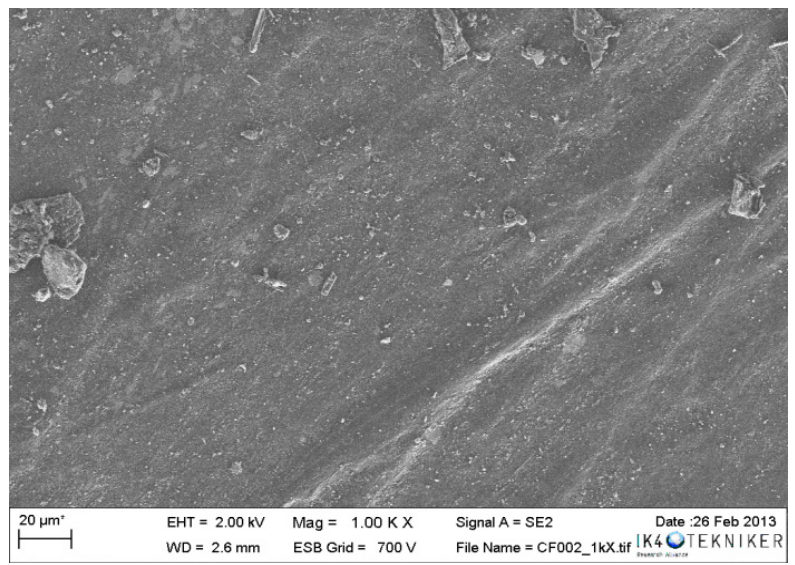

(a)

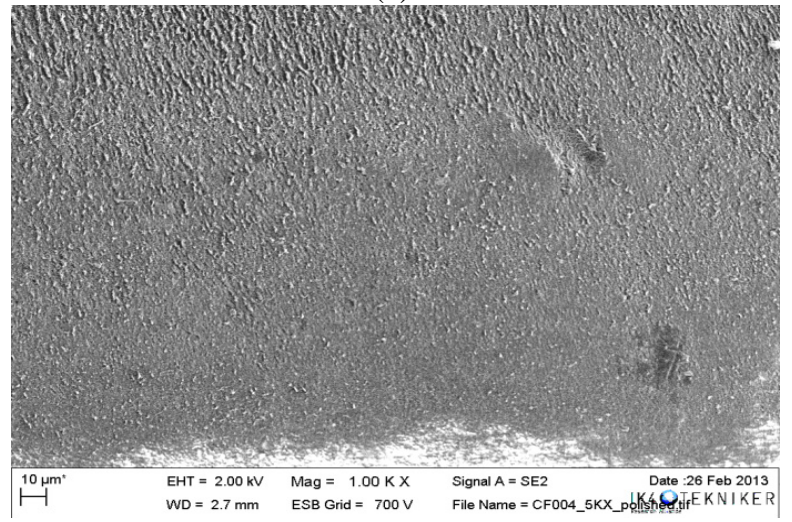

(b)

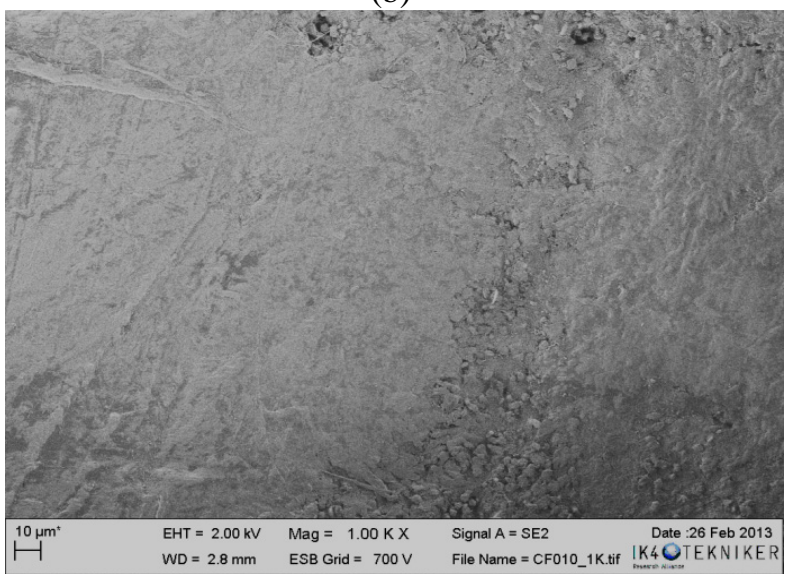

(c)

Figure 4: Samples' surfaces before the tribo-tests: a) NBR; b) TPU; c) PTFE. 
comparison with the contact length; the friction coefficient is known and variable during sliding. The work done by the friction force $\mathrm{Q}$ over the time can be expressed as

$$
Q(t)=\mu(t) \cdot W \cdot v
$$

where $\mu$ is the coefficient of friction, $\mathrm{W}$ the normal load and $\mathrm{v}$ the sliding velocity. The contact flash temperature based on Tian and Kennedy [4, 5] equations can be then described as function of the time by

$$
T_{\text {fmax }}(t)=\frac{2 \cdot Q(t)}{K \cdot A} \cdot \sqrt{\frac{2 \cdot \chi \cdot b}{\pi \cdot v}}
$$

where $\mathrm{K}$ is the thermal conductivity, $\chi$ the thermal diffusivity, $\mathrm{A}$ the contact surface and $2 \mathrm{~b}$ the contact width. It was not reliable to measure or to hypothesize the evolution of the contact area over time so the contact area considered for calculations in the Eqn. (2) is the one measured at the end of the test. Thus, the calculated temperatures were under-estimated in all the cases. Eqns (1) and (2), thus, describe the progressive evolution of the work done by the friction force and the temperature evolution at the contact.

Figure 5 shows the coefficient of friction and calculated contact flash temperature evolution over the time for the different studied polymers: the curves are the same excepting the multiplication by the geometrical dimensions of the contact and the thermal characteristics of the materials.

Results showed that the TPU presents a very high friction coefficient in comparison with the NBR and PTFE. During the running period, however, the NBR reaches friction coefficients as high as the TPU, probably because of its surface roughness ( $\mathrm{Ra} \approx 0.9$ ). The energy that is transformed as a consequence of frictional contact can be dissipated (conversion to heat, vibration, material

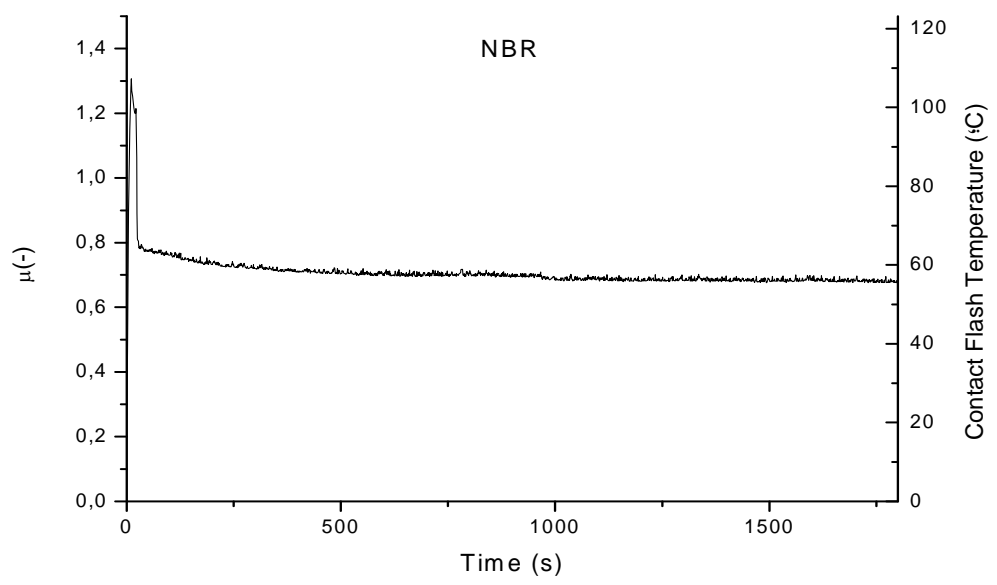

(a)

Figure 5: Coefficient of friction and calculated contact temperature evolution of polymeric samples. 


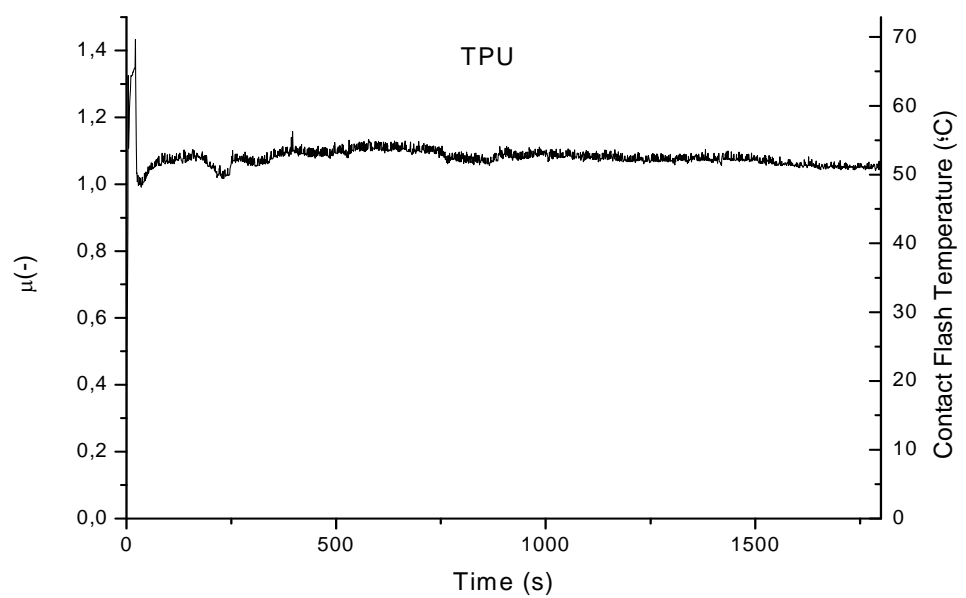

(b)

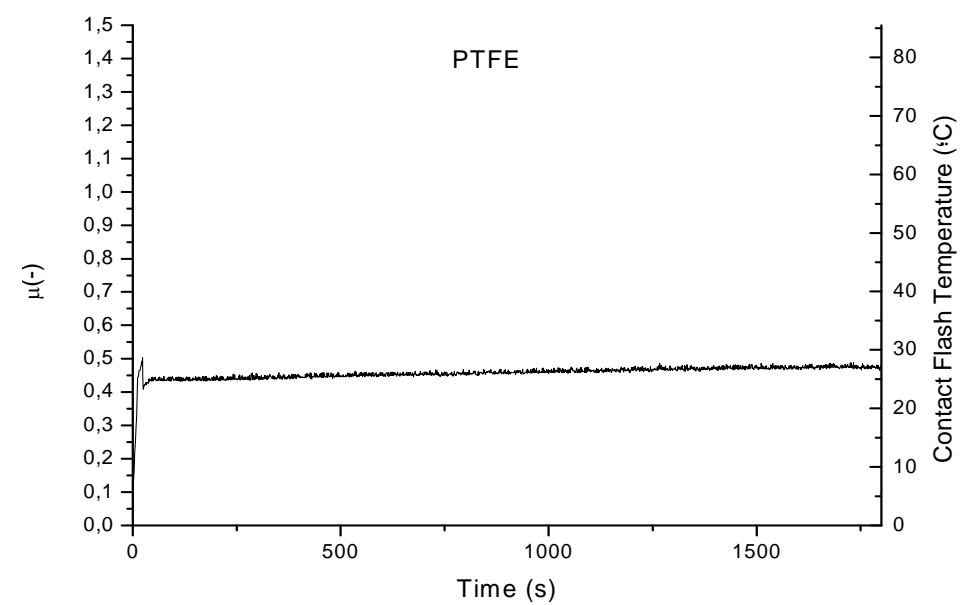

(c)

Figure 5: Continued.

deformation, new surface creation) or stored into the tribosystem. The work done by the friction i.e. the dissipated energy due to friction can be calculated as

$$
E=v \cdot W \cdot \int_{t i}^{t f} \mu(t) \cdot d t
$$

where $\mathrm{v}$ is the mean relative sliding velocity, $\mathrm{W}$ is the normal load, $\mu$ is the coefficient of friction, $t_{i}$ the initial time and $t_{f}$ the time at the end of the test. Frictional work values are presented in Table 1.

The resulting contact flash temperatures in NBR and TPU are very similar and closed to $55^{\circ} \mathrm{C}$, however, the resultant flash temperature values for the NBR during the running period are high enough (up to $107^{\circ} \mathrm{C}$ ) and close to the material limit of use. Wear scars of the three samples are very different. In Figure 6, the worn surfaces of the polymers after the tribological test can be observed. 
10 Surface Effects and Contact Mechanics XI

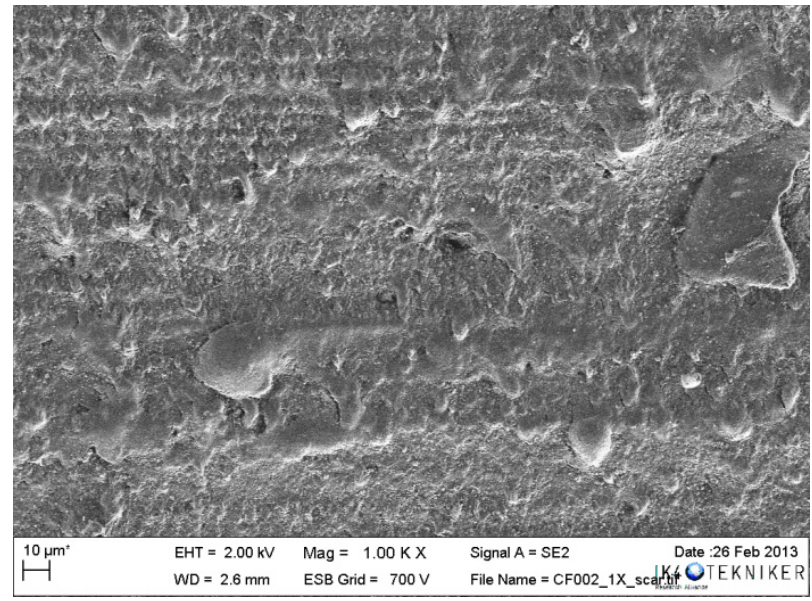

(a)

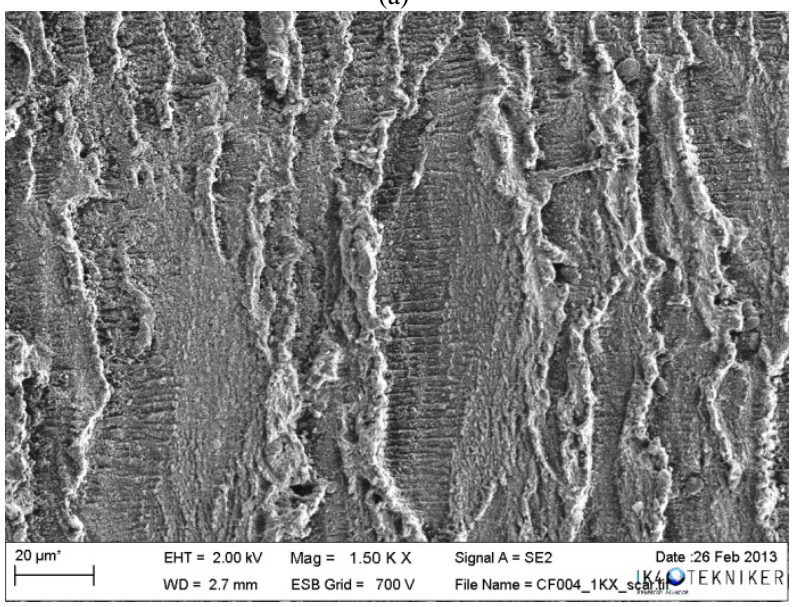

(b)

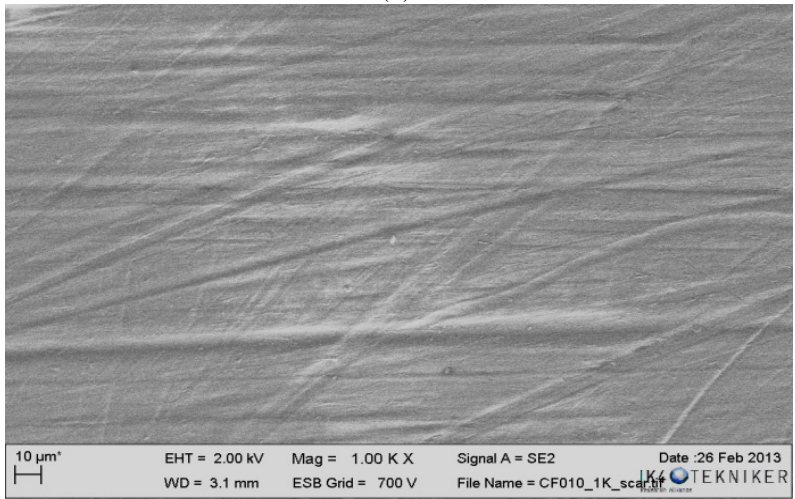

(c)

Figure 6: $\quad$ SEM of worn surfaces (a) NBR, (b) TPU, (c) PTFE. 
The wear properties of the rubber are mainly governed by its visco-elastic bulk properties, superimposed by surface effects of mainly thermo-chemical origin. The scar of the NBR sample has the edges more pronounced than the central part (Figure 7). Abrasion and blisters due to material transfer was observed i.e. the polymer was degraded under selected operating conditions. Although deciding which the main degradation mechanisms from a wear scar are is a difficult task, it is though that thermo-oxidation may happen since the NBR is quite susceptible to thermal and oxidative degradation (Degrange et al. [6]). The main wear mechanism for the TPU is usually the fatigue wear. This kind of fatigue wear, where the counterpart is smooth, as in this case that the $100 \mathrm{Cr} 6$ cylinder surface roughness is $\mathrm{Ra}=0.05$ and the friction coefficient is high enough, is known as frictional wear and the waves shown in Figure 6(b)) as fatigue waves (Martinez et al. [7]). In the case of virgin PTFE, polymer chains are oriented in the sliding direction. Although the wear was considerable the friction was low enough because the wear debris acted as a lubricant and the formation of a counter-surface layer avoid sliding against steel.

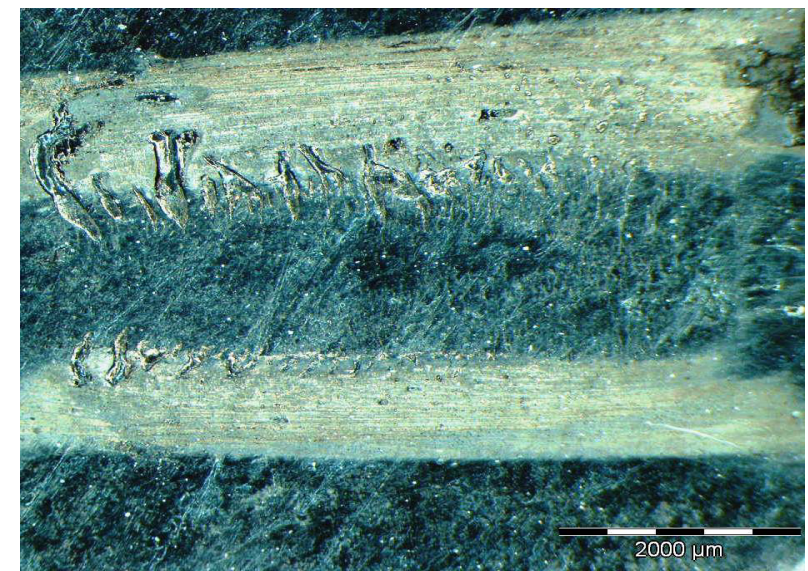

Figure 7: $\quad$ Worn surface of the NBR sample after the sliding tests.

\section{Conclusions}

In this work, in order to validate the calculation method developed in a previous study (Conte et al. [2]), it was applied to three polymers of completely different nature: Nitrile-Butadiene Rubber (NBR), Thermoplastic Polyurethane (TPU) and Polytetrafluoroethylene (PTFE). Results showed a good agreement with literature and previous studies for semi-crystalline materials like PTFE. Calculated maximum temperatures at the surface of the samples after the running in period showed quite similar values about $55^{\circ} \mathrm{C}$ for the NBR and TPU. For the case of the PTFE, calculations predicted temperatures in the range of $25-30^{\circ} \mathrm{C}$. The worn surfaces were investigated in order to link the calculated flash 
temperatures with the wear scars and TGA and DSC analysis results. The resultant scar in the NBR was so peculiar since abrasion and blisters due to material transfer was observed i.e. the polymer was degraded under selected operating conditions. Two hypotheses can be taken into consideration: the thermal degradation of the sample occurred as a consequence of the high temperatures, close to the material temperature limit reached during the runningin period of the tribo-test, as calculations showed or the frictional heating at the contact is higher than estimated analytically. Further investigations will be carried out using a thermo camera closed to the contact area.

\section{References}

[1] Conte M., Fernandez B., Igartua A.. Effect of surface temperature on tribological behavior of PTFE composites, $10^{\text {th }}$ International Conference on Surface Effects and Contact Mechanics, Malta, 2012.

[2] Conte M., Fernandez B., Igartua A.. Frictional heating calculations based on tailored experimental tests, $15^{\text {th }}$ NordTrib Conference, Trondheim, 2012.

[3] Friedrich K. Friction and wear of polymer composites. Composite Materials series, vol. 1, Elsevier, 1986.

[4] Kennedy F. E., Tian X. The effect of interfacial temperature on friction and wear of thermoplastics in the thermal control regime. Tribology Series 1994; 27 (C): 235-244.

[5] Stachowiak, G. W. and Batchelor, A. W., Engineering Tribology, Elsevier: Amsterdam, pp. 333-345, 2005.

[6] Degrange J.M., Thomine M., Lapsa Ph., Pelletier J.M., Chazeau L., Vigier G., Dudragne G., Guerbé L. Influence of viscoelasticity on the tribological behaviour of carbon black filled nitrile rubber (NBR) for lip seal application. Wear 259, pp. 687-692, 2005.

[7] F.J. Martinez, M. Canales, J. M. Bielsa, M. A. Jiménez. Relationship between wear rate and mechanical fatigue in sliding TPU-metal contacts. Wear 268, pp. 388-398, 2010. 